\title{
Distribución y ecología de algunas especies de rodófitos (Rhodophyta) en la cuenca del río Ebro
}

\author{
Pedro Tomás ${ }^{1, *}$, Jose Luis Moreno ${ }^{2}$, Marina Aboal $^{3}$, Javier Oscoz $^{4}$, Concha Durán $^{5}$, \\ Patricia Navarro ${ }^{5}$ y Andrea Elbaile ${ }^{1}$
}

${ }^{1}$ Laboratorio de Ensayos Técnicos S.A. ENSAYA. Pol. Ind. Valdeconsejo C/Aneto parcela 8-A, 50410 Cuarte de Huerva (Zaragoza).

${ }^{2}$ Centro Regional de Estudios del Agua (CREA), Universidad de Castilla-La Mancha. Ctra. de las Peñas, km 3, 02071 Albacete.

${ }^{3}$ Laboratorio de Algología. Departamento de Biología Vegetal. Facultad de Biología. Universidad de Murcia. Campus de Espinardo, 30100 Espinardo (Murcia).

${ }^{4}$ Departamento de Zoología y Ecología, Universidad de Navarra, Apdo. 177, 31080 Pamplona (Navarra).

5 Área de Calidad de Aguas, Confederación Hidrográfica del Ebro, Paseo de Sagasta 24-28, 50071 Zaragoza.

* Autor responsable de la correspondencia: biologia@ensaya.es

Recibido: 18/6/12

Aceptado: 11/2/13

\begin{abstract}
Distribution and ecology of some species of red algae (Rhodophyta) in the Ebro River Basin

The implementation of the Water Framework Directive (2000/60/CE) in the Ebro River Basin and the evaluation of the ecological status of rivers, lakes and wetlands are increasing the knowledge about the distribution and ecology of aquatic organisms, including the red algae (Rhodophyta). In this work we show new data concerning the distribution, habitat and environmental ranges of five species of Rhodophyta in the Ebro River Basin with scarce records: Bangia atropurpurea, Chroodactylon ornatum, Chroothece rupestris, Compsopogon coeruleus and Thorea hispida. The former three species showed an optimum development in calcareous mountain streams while the latter two species did in lower reaches where salinity and eutrophication increase.
\end{abstract}

Key words: Algae, Rhodophyta, Ebro, environmental ranges, geographic distribution.

\section{RESUMEN}

Distribución y ecología de algunas especies de rodófitos (Rhodophyta) en la cuenca del río Ebro

La aplicación de la Directiva Marco del Agua (2000/60/CE) en la cuenca del Ebro y la consiguiente evaluación del estado ecológico de los ríos, lagos y humedales mediante indicadores biológicos (red CEMAS, Control del Estado de las Masas de Agua Superficiales), están permitiendo aumentar el conocimiento de la distribución y ecología de los organismos acuáticos, entre ellos las algas rojas (Rhodophyta). Fruto de este seguimiento, se presentan nuevos datos sobre la distribución, hábitat y ecología de cinco especies de rodófitos en la cuenca del Ebro de las que existen datos escasos: Bangia atropurpurea, Chroodactylon ornatum, Chroothece rupestris, Compsopogon coeruleus y Thorea hispida. Los resultados indican que las tres primeras tienen un desarrollo óptimo en ríos calcáreos de montaña, mientras que las dos últimas lo tienen en tramos medios y bajos donde aumenta la salinidad y la concentración de nutrientes.

Palabras clave: Algas, Rhodophyta, Ebro, rangos ambientales, distribución geográfica. 


\section{INTRODUCCIÓN}

Los rodófitos son un grupo de algas amenazado en Europa, propio de aguas blandas, claras y puras, siendo, en general, indicadores de buena calidad ecológica (Eloranta \& Kwandrans, 2004). En el norte de Europa los rodófitos son frecuentes y abundantes, mientras que en el centro y sur de Europa son más escasos, reduciendo su distribución conocida a áreas montañosas donde los impactos humanos como la eutrofización y la regulación hidrológica mediante embalses, presas y canalizaciones son menores (Kwandrans \& Eloranta, 2010). Debido a su ciclo de vida largo y complejo (Lee, 1980; van den Hoek et al., 1995), necesitan unas condiciones ambientales hidrológicamente estables, y son en general débiles competidores: en tramos altos compiten con los briófitos por el sustrato, mientras que en tramos medios y bajos, de aguas más eutróficas y turbias, compiten con los clorófitos filamentosos y las cianobacterias (Eloranta \& Kwandrans, 2004). La predominancia de pigmentos como ficoeritrina y ficocianina les confiere colores muy llamativos como el rojo, violeta, azul, verde, marrón o grisáceo.

Desde la publicación del catálogo nacional de rodófitos (Álvarez-Cobelas, 1984), las aportaciones al conocimiento de este grupo algal han sido escasas y las más destacadas se publicaron hace dos décadas (p. ej. Llimona et al., 1985; Aboal, 1989; Sabater et al., 1989). Tampoco existen estudios extensivos previos sobre este grupo de algas que cubran toda la cuenca del Ebro. Durante los últimos años, la obligación de cumplir con los objetivos establecidos en la Directiva Marco del Agua (DMA) (Directiva 2000/60/CE) y su aplicación en los ríos de la Península Ibérica, ha dado lugar a un incremento del conocimiento de los diferentes componentes bióticos de nuestros ríos: macrófitos (macroalgas, briófitos y plantas vasculares), macroinvertebrados, diatomeas, peces y vegetación de ribera. Por otra parte, este seguimiento continuo durante varios años de la biota que habita nuestros ríos está permitiendo analizar variaciones temporales (estacionales e interanuales) en la distribución espacial de los diferentes organismos acuáticos, así como ampliar los rangos ecológicos de los mismos.
En relación con la flora acuática, en la cuenca del Ebro se viene aplicando el índice trófico denominado IVAM (Moreno et al. 2006), con el fin de evaluar el estado ecológico de los ríos. Este índice utiliza los organismos acuáticos autótrofos y macroscópicos identificados a nivel de género, entre los que se incluyen las algas rodófitas. Sin embargo, a nivel específico no se han realizado estudios recientes sobre la distribución y ecología de los rodófitos en la cuenca del Ebro, por lo que la aportación de nuevos datos sobre la presencia de especies de rodófitos resulta de gran interés florístico y ecológico.

En el presente trabajo se presenta la distribución, el hábitat y los rangos ambientales de algunas especies de rodófitos sobre las que existen escasas citas en la cuenca del Ebro o en la Península Ibérica: Bangia atropurpurea, Chroodactylon ornatum, Chroothece rupestris, Compsopogon coeruleus y Thorea hispida.

\section{MATERIAL Y MÉTODOS}

Durante los meses de mayo a septiembre en el periodo 2006-2011, se visitaron entre 211 (2006) y 362 (2009) estaciones de muestreo pertenecientes a las redes de control operativo, vigilancia y referencia de las masas de agua de la cuenca del Ebro, localizada en el noreste peninsular. Para ello, se seleccionaron tramos representativos de unos $100 \mathrm{~m}$ de longitud, donde se realizaron recorridos en zigzag aguas arriba y de orilla a orilla, recolectando todos los macrófitos visibles a simple vista. La abundancia de cada taxon se registró como el porcentaje de cobertura en el tramo de muestreo. De acuerdo con la metodología adoptada para el seguimiento del estado ecológico en las cuencas hidrográficas españolas, la mayoría de los muestreos fueron realizados entre finales de primavera y verano (Anexo I, material adicional disponible en www.limnetica.net/internet). El número de puntos de muestreo fue establecido siguiendo los criterios de delimitación de masas de agua definidos para la Directiva ("Identification of Water Bodies", Guidance document n ${ }^{\circ}$ 2, Common Implementation Strategy for the Water Framework Directive), de forma que exista al menos un pun- 
to de control por cada masa de agua. Según la regionalización fisiográfica oficial de los ríos españoles realizada por el Centro de Estudios Hidrográficos (CEDEX), los ríos de la cuenca del Ebro pertenecen a las siguientes tipologías: ríos mineralizados de baja montaña mediterránea (código 109); ríos de montaña mediterránea calcárea (112); ejes mediterráneo-continentales poco mineralizados (115); ejes mediterráneo-continentales mineralizados (116); grandes ejes en ambiente mediterráneo (117); ríos de montaña mediterránea silícea (111); ríos de montaña húmeda calcárea (126) y ríos de alta montaña (127).

Los organismos fueron fijados en formol al $4 \%$ y se llevaron al laboratorio para ser analizados con la lupa binocular y el microscopio (CHE, 2005). En algunos casos (Chroothece) se tomaron muestras de limos y areniscas por encima del nivel del agua y se humedecieron para detectar la presencia de estas algas. Además del muestreo biológico, en cada visita se midieron algunos parámetros fisicoquímicos in situ mediante una sonda multiparamétrica YSI 556 MPS (pH, temperatura, conductividad y oxígeno disuelto) y se tomaron muestras de agua para analizar posteriormente en el laboratorio la concentración de nitrato, amonio y fosfato (fósforo reactivo soluble, PRS). Los análisis de agua se realizaron según los métodos y protocolos ITC-MMA EECC-1/06 (MMA, 2006). Una vez realizado el muestreo de cada una de las estaciones, se procedió a la desinfección del material y de los equipos de muestreo utilizados, de acuerdo con el protocolo establecido por la CHE (2007) para evitar la propagación del mejillón cebra y otros organismos. Las fotografías se realizaron con un microscopio Olympus BX50 equipado con un set Colorview II. Una parte del material se prensó y se conservó en pliegos de herbario que se depositaron en el herbario de la Universidad de Murcia (MUB-ALGAS).

Los rangos ambientales y preferencias ecológicas de las especies se describen mediante gráficos comparativos de cajas y líneas. Con el fin de comprobar estadísticamente las tendencias ambientales observadas, se realizó un análisis de correlaciones de Spearman entre las variables ambientales estandarizadas y los datos de presencia-ausencia de las especies. Los valores de los pa- rámetros ambientales pertenecen a los datos oficiales de las redes de control biológico de la Confederación Hidrográfica del Ebro correspondientes a los años 2006-2011, así como a otras observaciones esporádicas realizadas en 2011 y 2012.

\section{RESULTADOS}

Durante el periodo de estudio se tomaron y procesaron en total 1350 muestras de macrófitos acuáticos, 264 de las cuales presentaron alguna de las 5 especies de rodófitos analizadas. En el Anexo I (www.limnetica.net/internet) se enumeran las localidades donde fueron recolectadas junto con la fecha de muestreo, abundancia, coordenadas UTM, la tipología fluvial oficial CEDEX y la provincia administrativa.

\section{Distribución y hábitat}

Bangia atropurpurea (Roth 1806) C. Agardh 1824 (Fig. 1) se recolectó en diferentes tipos de ríos, desde la alta montaña en los Pirineos hasta los tramos medios de algunos afluentes principales del Ebro, especialmente en las cuencas del Cinca y Jalón y sobre sustratos muy diversos: Cladophora sp., Oedogonium sp., Potamogeton pectinatus, musgos, piedras, roca madre, cemento de puentes, hilos de pescar, plásticos o tallos de Phragmites australis y Rubus sp., todos ellos presentes en zonas turbulentas y de elevada corriente. La profundidad osciló entre los 0 y $20 \mathrm{~cm}$. En algunos tramos, como en el río Ésera en Graus, se ha observado sobre el mismo sustrato y lugar en años sucesivos.

Chroodactylon ornatum (C. Agardh) Basson 1979 es en la cuenca del Ebro una especie común en ríos calcáreos de montaña. Se ha detectado principalmente creciendo de forma epífita sobre Cladophora sp., sobre todo en talos con deposiciones de carbonatos y con presencia de cianobacterias como Lyngbya sp. y Chamaesiphon sp., así como de diatomeas (Cocconeis sp.). Con menor frecuencia, se ha observado creciendo sobre musgos, Vaucheria sp., Oedogonium sp., Lemanea sp., limos y fundas de tricópteros de la familia Hydroptilidae. 

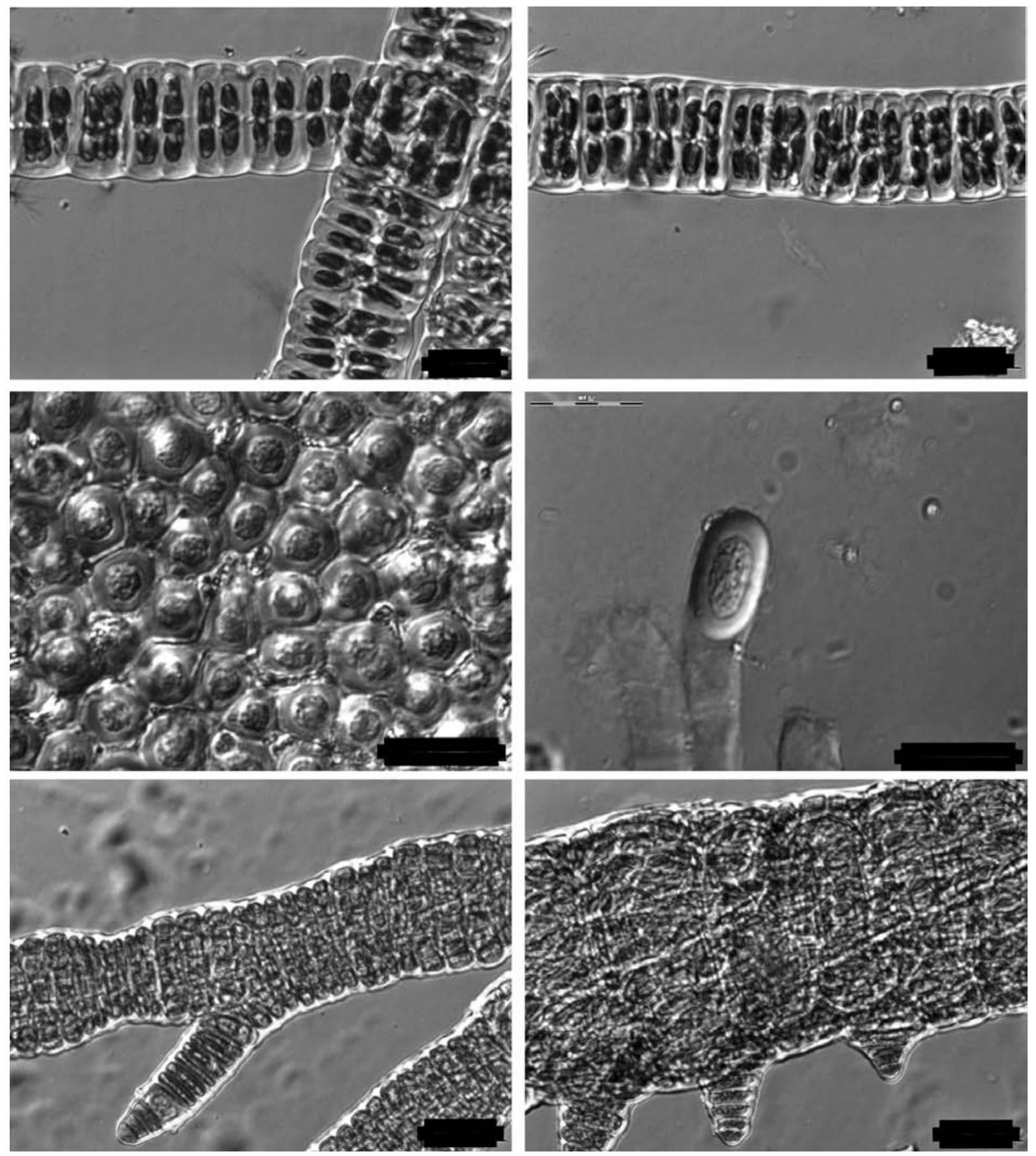

Figura 1. Arriba: filamentos multiseriados de Bangia atropurpurea en el río Alcanadre. Centro: vista superficial de una colonia de Chroothece rupestris y detalle de una célula con su pedúnculo en el río Arba de Riguel en Sádaba. Abajo: detalle de la corticación y de las ramas de Compsopogon coeruleus en el río Ebro en Pina de Ebro. La escala representa $50 \mu \mathrm{m}$ en las imágenes de arriba y centro y $20 \mu \mathrm{m}$ abajo. Up: multiseriate filaments of Bangia atropurpurea in the Alcanadre River. Center: surface view of a colony of Chroothece rupestris and detail of a cell with its stem in the Arba de Riguel River in Sádaba. Down: detail of the cortication and the branches of Compsopogon coeruleus in the Ebro river at Pina de Ebro. The scale represents 50 microns (up and center) and 20 microns (down). 

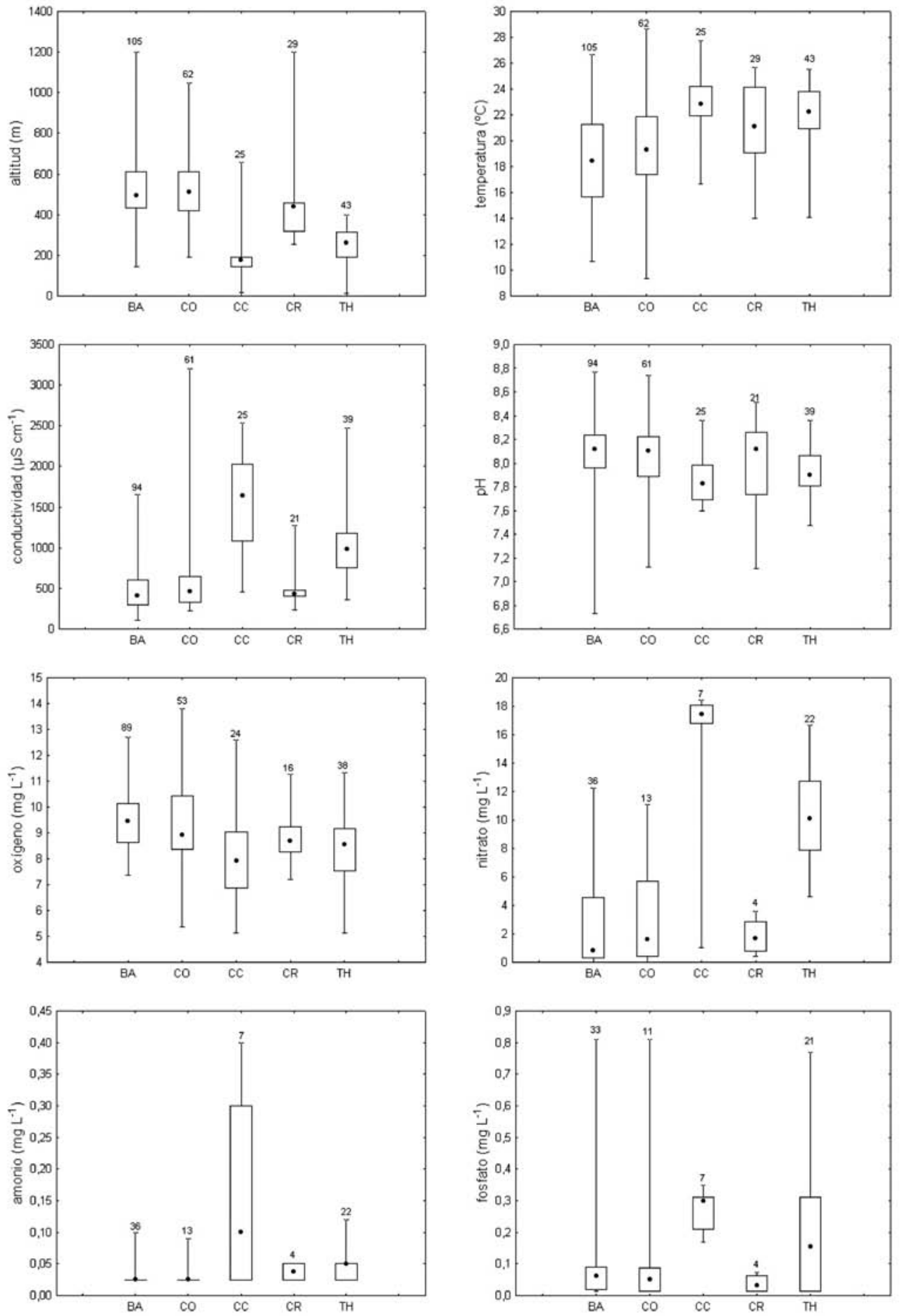

Figura 2. Gráficos comparativos de cajas y líneas de las cinco especies estudiadas para las ocho variables ambientales medidas. Los números encima de las líneas hacen referencia al número de casos $(n)$. Los puntos representan la mediana; las cajas el $25 \%$-75\%; y la barra el Mín-Máx. BA: Bangia atropurpurea; CO: Chroodactylon ornatum; CC: Compsopogon coeruleus; CR: Chroothece rupestris; TH: Thorea hispida. Comparative Box and whiskers graphs of the five studied species for the eight environmental variables measured. Numbers above whiskers mean the number of cases (n). Dots represent the median; boxes the 25\%-75\%; and bars Min-Max. BA: Bangia atropurpurea; $C O$ : Chroodactylon ornatum; $C C$ : Compsopogon coeruleus; $C R$ : Chroothece rupestris; $T H$ : Thorea hispida. 
Chroothece rupestris Hansgirg 1884 (Fig. 1) presentó una distribución reducida en la cuenca del Ebro. Se ha recolectado principalmente en ríos mineralizados de baja montaña calcárea, especialmente en los ríos Flumen y Alcanadre. Esta especie se encontró creciendo en sustratos diversos: sobre piedras sumergidas (epilítica), epífita de musgos y en taludes de las márgenes del río entre 15 y $50 \mathrm{~cm}$ por encima del nivel del agua. En general, las colonias presentaron formas semiesféricas o en tapetes, con un tamaño que osciló entre $3 \mathrm{~mm}$ y $3 \mathrm{~cm}$.

Compsopogon coeruleus (Balbis) Montagne 1850 (Fig. 1) se halló en el eje principal del río Ebro y en tramos medios de los ríos Jalón (aguas abajo de la localidad de Alhama de Aragón, tramo con influencia de surgencias termales), Martín y Segre. Estos tramos presentaron un caudal elevado y una profundidad de la lámina de agua superior a los $50 \mathrm{~cm}$. En el tramo medio del río Ebro, las aguas tuvieron una turbidez elevada durante la mayor parte del periodo de estudio, siendo difícil su visualización. Sin embargo, durante el año 2011, con caudales bajos y aguas más transparentes que en años anteriores, la especie se recolectó en nuevas localidades. Los talos filamentosos se encontraron creciendo sobre piedras del fondo entremezclados con Cladophora sp., o epífitos sobre Cladophora sp., Potamogeton pectinatus y Myriophyllum sp.

Thorea hispida (Thore) Desvaux 1818 presentó una distribución restringida, limitándose al río Ebro y a los tramos bajos de los ríos Arga, Aragón y Gállego. La especie prefiere aguas turbias de profundidad variable (desde $10 \mathrm{~cm}$ hasta más de $1 \mathrm{~m})$ y se encontró creciendo sobre bloques y piedras cubiertas de sedimento, así como sobre el molusco invasor Dreissena polymorpha. En los años 2008 y 2011 la especie fue más frecuente, recolectándose ejemplares de longitud superior a $1 \mathrm{~m}$. En ejemplares de longitud superior a $1.5 \mathrm{~cm}$, se observaron estructuras reproductoras, detectándose la fase Chantransia sobre bloques y también epífita de Potamogeton pectinatus.

Respecto a la abundancia de las especies, en la mayoría de los casos cubrieron menos del $0.1 \%$ de cauce, excepto en cinco ocasiones: $B$. atropurpurea alcanzó una cobertura del $50 \%$ en el río Alcanadre en junio de 2010, mientras que $C$. rupestris cubrió entre el 1 y el $5 \%$ del cauce en cuatro ocasiones, tres en junio (río Alcanadre en 2010 y 2011; río Arba de Riguel en 2009) y una en febrero (río Alcanadre) (Anexo 1. www.limnetica.net/internet).

\section{Preferencias ecológicas}

En la figura 2 se presentan los valores máximos y mínimos, así como el rango de óptimo desarrollo de las especies en la cuenca del Ebro, obtenidos durante el periodo de estudio para las 8 variables fisicoquímicas estudiadas (el intervalo comprendido entre los percentiles 25 y 75 incluye los valores ambientales medidos con más frecuencia para cada especie). B. atropurpurea, $C$. ornatum y $C$. rupestris son, en la cuenca del Ebro, especies típicas de montaña, con presencia en altitudes superiores a $1000 \mathrm{~m}$ y desarrollo óptimo entre 300-600 m, mientras que $C$. coeruleus y $T$. hispida se presentaron típicamente en tramos medios y bajos entre 100-300 m de altitud. Los valores de correlación entre las especies y la altitud fueron estadísticamente significativos excepto para $C$. rupestris, lo que indica una clara influencia de la altitud en la frecuencia de aparición de dichas especies (Tabla 1). De la misma forma, B. atropurpurea, C. coeruleus y T. hispi$d a$ mostraron correlaciones significativas con la temperatura, la primera encontrando su óptimo en aguas más frías que las dos últimas.

En cuanto a la salinidad, todas las especies presentaron un rango amplio de tolerancia, pudiendo desarrollarse en aguas con valores de conductividad elevados, superiores a $1000 \mu \mathrm{S} \mathrm{cm}^{-1}$. No obstante, el rango óptimo de desarrollo de $B$. atropurpurea, $C$. ornatum y $C$. rupestris se encuentra en torno a los $500 \mu \mathrm{S} \mathrm{cm}^{-1}$ de conductividad, mientras que $C$. coeruleus y $T$. hispida prefieren aguas con cierta salinidad, entre 1000-2000 $\mu \mathrm{S} \mathrm{cm}^{-1}$. Estas dos especies mostraron correlaciones positivas y significativas con la conductividad del agua (más frecuentes en aguas salinas), mientras que B. atropurpurea presentó una correlación negativa significativa (más frecuente en aguas dulces) (Tabla 1).

Respecto al $\mathrm{pH}$, las cinco especies se desarrollaron óptimamente en aguas básicas, siendo $C$. 
Tabla 1. Coeficientes de correlación de Spearman entre los parámetros ambientales y las especies de rodófitos estudiadas (***: $p<0.001, * *: p<0.01 ; *: p<0.05)$. Spearman's correlation coefficient between environmental parameters and species $(* * *:$ $\mathrm{p}<0.001, * *: \mathrm{p}<0.01 ; *: \mathrm{p}<0.05)$.

\begin{tabular}{|c|c|c|c|c|c|c|c|c|c|}
\hline & $\begin{array}{c}\text { Bangia } \\
\text { atropurpurea }\end{array}$ & & $\begin{array}{c}\text { Chroodactylon } \\
\text { ornatum }\end{array}$ & & $\begin{array}{c}\text { Compsopogon } \\
\text { coeruleus }\end{array}$ & & $\begin{array}{c}\text { Chroothece } \\
\text { rupestris }\end{array}$ & $\begin{array}{l}\text { Thorea } \\
\text { hispida }\end{array}$ & \\
\hline altitud & 0.3585 & $* * *$ & 0.2491 & $* * *$ & -0.3649 & $* * *$ & $\mathrm{~ns}$ & -0.4676 & $* * *$ \\
\hline temperatura & -0.3240 & $* * *$ & ns & & 0.2820 & $* * *$ & ns & 0.2562 & $* * *$ \\
\hline $\mathrm{pH}$ & 0.2853 & $* * *$ & ns & & -0.2550 & $* * *$ & ns & -0.2274 & $* * *$ \\
\hline conductividad & -0.3356 & $* * *$ & -0.1379 & $*$ & 0.4113 & $* * *$ & ns & 0.3623 & $* * *$ \\
\hline oxígeno & 0.2678 & $* * *$ & ns & & -0.2069 & $* *$ & ns & -0.1979 & $* *$ \\
\hline amonio & -0.3388 & $* *$ & ns & & 0.2212 & $*$ & $\mathrm{~ns}$ & 0.3429 & $* *$ \\
\hline nitrato & -0.5160 & $* * *$ & ns & & 0.3997 & $* * *$ & ns & 0.5023 & $* * *$ \\
\hline fosfato & ns & & ns & & 0.3567 & $* *$ & $\mathrm{~ns}$ & ns & \\
\hline
\end{tabular}

coeruleus y $T$. hispida más frecuentes en aguas con los valores de $\mathrm{pH}$ más bajos medidos. De hecho, ambas especies mostraron correlaciones negativas significativas con este parámetro (Tabla 1). Los valores óptimos de oxígeno disuelto para las cinco especies se encontraron por encima de $8 \mathrm{mg} \mathrm{L}^{-1}$, si bien $C$. ornatum, $C$. coeruleus y $T$. hispida se han recolectado ocasionalmente en aguas con valores bajos, entre 5-6 $\mathrm{mg} \mathrm{L}^{-1}$. C. coeruleus y $T$. hispida presentaron correlaciones negativas significativas con el oxígeno disuelto, indicando su preferencia por aguas menos oxigenadas.

En cuanto a las condiciones tróficas, todas las especies, excepto $C$. rupestris, han sido recolectadas en aguas eutróficas en algún momento del periodo de estudio, lo que indica la tolerancia de estos rodófitos a la eutrofización (Fig. 2). C. coeruleus es la especie que presentó un desarrollo óptimo en las aguas más eutróficas, tolerando frecuentemente concentraciones de nitrato superiores a $16 \mathrm{mg} \mathrm{L}^{-1}$, entre $0.1-0.3 \mathrm{mg} \mathrm{L}^{-1}$ de amonio y entre $0.2-0.3 \mathrm{mg} \mathrm{L}^{-1}$ de fosfato, y presentando además correlaciones positivas significativas con los tres nutrientes medidos (Tabla 1). En el extremo opuesto se encuentra $B$. atropurpurea, que mostró correlaciones negativas significativas con la concentración de nitrato y amonio, indicando su preferencia por las aguas oligomesotróficas. C. ornatum y $C$. rupestris presentaron también un desarrollo óptimo en aguas oligomesotróficas (Fig. 2), aunque no se obtuvieron correlaciones significativas con ninguno de los nutrientes analizados (Tabla 1). Finalmente, T. hispida presentó una alta correlación significativa con aguas ricas en nitrato, y aunque su correlación con el fosfato no fue significativa, se trata de una especie con desarrollo óptimo en aguas eutróficas con altos niveles de nitrato y fosfato (Fig. 2).

\section{DISCUSIÓN}

Bangia atropurpurea es una especie de aguas salobres y marinas, pero que ha invadido las aguas continentales como grandes ríos, canales y lagos de alta conductividad (Eloranta \& Kwandrans, 2002; Sheath \& Sherwood, 2002; Kwandrans \& Eloranta, 2010). En España se trata de una especie escasamente citada, mientras que en Europa es un rodófito común en tramos altos de montaña (Sabater et al., 1989). Nuestros resultados indican que en la cuenca del Ebro se trata de una especie frecuente, ya que ha sido recolectada en 66 localidades. En la cuenca del Ebro es propia de aguas dulces de montaña oligomesotróficas, aunque puede tolerar altos niveles de eutrofia en tramos inferiores (>0.8 $\mathrm{mg} \mathrm{L}^{-1}$ de fosfato).

Según Sheath (2003) Chroothece es un raro componente de arroyos, suelos húmedos y turberas. C. rupestris ocupa hábitats y sustratos diversos como rocas húmedas de aguas alcalinas en ambientes poco iluminados (Starmach, 1977; Eloranta et al., 2011), superficies higropétricas de fuentes (Margalef, 1955) o epilíticas y sumergidas en arroyos alcalinos de corriente débil (Aboal, 1989; Sabater et al., 1989). Se trata de una especie cuya distribución es poco conocida 
en la península, por lo que se considera de interés su ampliación a 19 localidades más. Es conveniente comentar que la clave más actual disponible para la diferenciación entre las especies $C$. rupestris y $C$. richtheriana (Eloranta et al., 2011) utiliza el tamaño celular como carácter diagnóstico principal (15-18 $\mu \mathrm{m}$ para $C$. richtheriana y 9-15 $\mu \mathrm{m} C$. rupestris) y secundariamente el hábitat (aguas algo salinas y sustratos limosos para $C$. richtheriana; aguas dulces y sustratos duros para $C$. rupestris). Las poblaciones estudiadas en la cuenca del Ebro presentaron un tamaño entre 10 y $25 \mu \mathrm{m}$, lo que incluiría a ambas especies, pero de acuerdo con los valores de conductividad propios de los arroyos calcáreos $\left(485 \mu \mathrm{S} \mathrm{cm} \mathrm{cm}^{-1}\right.$ de valor medio, con un rango de 236-1268 $\mu \mathrm{S}$ $\mathrm{cm}^{-1}$ ) y el tipo de hábitat (sumergida, epilítica) indica que se trataría de $C$. rupestris. Estas características biométricas y ecológicas de las poblaciones de la cuenca del Ebro son similares a las poblaciones de $C$. rupestris citadas para arroyos alcalinos de bajo caudal del norte y sur peninsular (9-20 $\mu \mathrm{m}$ de longitud celular y 250$1100 \mu \mathrm{S} \mathrm{cm}^{-1}$ de conductividad; Sabater et al., 1989; Aboal, 1989).

En el área de estudio, el epifitismo de Chroodactylon ornatum sobre Cladophora sp., los géneros epífitos acompañantes, así como los rangos ambientales, coinciden con lo indicado para distintas zonas geográficas (Starmach, 1966; Sheath \& Morrison, 1982; Sheath \& Sherwood, 2002; Wolowski et al., 2007). En la Península Ibérica es una especie eurioica que crece en aguas dulces o salinas (hasta $25000 \mu \mathrm{S} \mathrm{cm}^{-1}$ ), tanto oligotróficas como eutróficas (Aboal 1989; Sabater et al. 1989). En la cuenca del Ebro se ha recolectado principalmente en arroyos de agua dulce, aunque también ocasionalmente en tramos más salinos y eutróficos.

Compsopogon coeruleus es una especie ampliamente distribuida en arroyos cálidos templados y tropicales (Kwandrans \& Eloranta, 2010). En la Península Ibérica las citas son escasas, creciendo principalmente en aguas salobres cerca de la costa o en ramblas salinas del sureste peninsular (Aboal, 1989; Sabater et al., 1989), y ocasionalmente en aguas dulces interiores (Busquets et al., 1985). En la cuenca del Ebro, C. coeruleus se ha recolectado en 16 localidades, correspon- diendo con tramos medios caudalosos de aguas cálidas, alcalinas, mineralizadas y eutróficas.

En general, T. hispida es una especie que crece en ríos y arroyos europeos con aguas turbias y eutróficas, sobre piedras o arena (Eloranta \& Kwandrans, 2007). Dos localidades ibéricas han sido previamente citadas para la especie: el Marjal de Pego-Oliva, Valencia (Tomás, 1981) y el río Ebro, Tarragona (Sabater et al., 1989). En el Marjal de Pego-Oliva, Egidos \& Aboal (2003) citaron Thorea violacea en una surgencia de aguas subsalinas y cálidas $\left(3500 \mu \mathrm{S} \mathrm{cm} \mathrm{cm}^{-1}, 21.3^{\circ} \mathrm{C}\right)$ y posteriormente se recolectó T. hispida en las partes bajas del río Bullent (Aboal, dat. inédt.). En el presente estudio se amplía su distribución a 22 sitios más, cuyas condiciones ambientales de aguas cálidas, alcalinas, bien oxigenadas, de conductividad elevada y alta concentración de nutrientes coinciden con las indicadas previamente por otros autores (Tomás et al., 1980; Sabater et al., 1989; Rott et al., 1999; Eloranta \& Kwandrans, 2007).

\section{AGRADECIMIENTOS}

Los autores están muy agradecidos a todas las personas que han colaborado en los diferentes muestreos que se han llevado a cabo en la cuenca del Ebro en los últimos años. En especial a Andrés Mellado, Isidoro Pérez, José Miguel García, Pablo Jáimez-Cuéllar, Julio M. Luzón y José Antonio Palomino por su trabajo en los muestreos de la cuenca del Ebro en el periodo 2006-2009.

\section{BIBLIOGRAFÍA}

ABOAL, M. 1989. Contribución al conocimiento de las algas epicontinentales del SE de España II: Los rodófitos (Rhodophyceae). Lazaroa, 11: 115-122.

ÁLVAREZ-COBELAS, M. 1984. Catálogo de las algas continentales españolas. II. Craspedophyceae. Cryptophyceae, Chrysophyceae, Dinophyceae, Euglenophyceae, Haptophyceae, Phaeophyceae, Rhodophyceae, Xanthophyceae. Acta Botanica Malacitana, 9: 27-40.

BUSQUETS, J., M. C. PICADO \& M. C. HERNÁNDEZ-MARINÉ. 1985. Compsopogon coeruleus 
(Balbis) Montagne (Rhodophyta, Bangiophyceae). Ampliación de su área de distribución en la Península Ibérica. Collectanea Botanica, 16(1): 229-230.

CHE (Confederación Hidrográfica del Ebro) 2005. Metodología para el establecimiento del Estado Ecológico según la Directiva Marco del Agua: Protocolos de muestreo y análisis. Ministerio de Medio Ambiente.

CHE (Confederación Hidrográfica del Ebro). 2007. Protocolo de desinfección de embarcaciones y equipos en masas de agua infectadas por mejillón cebra (Dreissena polymorpha). Ministerio de Medio Ambiente y Medio Rural y Marino.

EGIDOS, A. I. \& M. ABOAL. 2003. Thorea violacea Bory de Saint-Vicent (Thoreaceae, Rhodophyceae) en una surgencia del Marjal de Pego-Oliva, Comunidad Valenciana. Nueva cita para la flora algal española. Anales del Jardín Botánico de Madrid, 60(1): 27-32.

ELORANTA, P. \& J. KWANDRANS. 2002. Notes on some interesting freshwater Rhodophyta from Finland. Algological Studies, 105: 95-109.

ELORANTA, P. \& J. KWANDRANS. 2004. Indicator value of freshwater red algae in running Waters for water quality assessment. Oceanological and Hydrobiological Studies, International Journal of Oceanography and Hydrobiology, 33(1): 47-54.

ELORANTA, P. \& J. KWANDRANS. 2007. Freshwater Red Algae (Rhodophyta): Identification Guide to European Taxa, Particularly to Those in Finland. Norrlinia, 15: 1-103.

ELORANTA, P., J. KWANDRANS \& E. KUSELFETZMANN. 2011. Süsswasserflora von Mitteleuropa, Bd 7: Rhodophyta and Phaeophyceae. Spektrum Akademischer Verlag. 155 pp.

KWANDRANS, J. \& P. ELORANTA. 2010. Diversity of freshwater red algae in Europe. International Journal of Oceanography and Hydrobiology, 39: 161-169.

LEE, R. E. 1980. Phycology. Cambridge University Press, Cambridge.

LLIMONA, X., E. BALLESTEROS, M. BRUGUÉS, F. A. COMÍN, R. M. CROS, J. MOLERO, J. ROMERO, X. TOMÁS \& F. TORRELLA. 1985. Història Natural dels Països Catalans: Plantes Inferiors. Tom 4. Enciclopèdia Catalana, S. A, Barcelona.

MARGALEF, R. 1955. Materiales para una flora de las algas del NE de España. VI. Desmidiales, Rhodophyceae. Collectanea Botanica, 4: 319-330.
MMA (Ministerio de Medio Ambiente). 2006. ORDEN MAM/3207/2006, de 25 de septiembre, por la que se aprueba la instrucción técnica complementaria MMA-EECC-1/06, determinaciones químicas y microbiológicas para el análisis de las aguas. ITC-MMA, EECC-1/06. BOE, 250: 3632636440.

MORENO, J. L., C. NAVARRO \& J. DE LAS HERAS. 2006. Propuesta de un índice de vegetación acuática (IVAM) para la evaluación del estado trófico de los ríos de Castilla-La Mancha: Comparación con otros índices bióticos. Limnetica, 25 (3): 821-838.

ROTT, E., P. PFISTER, H. VAN DAM, K. PALL, E. PIPP, N. BINDER \& K. ORTLER. 1999. Indikationslisten für Aufwuchsalgen. Teil 2: Trophieindikation und autökologische Anmerkungen, Bundesministerium für Land- und Forstwirtschaft. Wasserwirtschaftkataster.

SABATER, S., M. ABOAL \& J. CAMBRA. 1989. Nuevas observaciones de rodofíceas en aguas epicontinentales del NE y SE de España. Limnetica, 5: 93-100.

SHEATH, R. G. 2003. Red Algae. In: Freshwater Algae of North America. John D. Wehr \& Robert G. Sheath (eds.): 197-224. Elsevier Science, San Diego, USA.

SHEATH, R. G. \& M. O. MORISON. 1982.Epiphytes on Cladophora glomerata in the Great Lakes and St. Lawrence Seaway with particular reference to the red alga Chroodactylon ramosum (= Asterocytis smargdina). Journal of Phycology, 18: 385-391.

SHEATH, R. G. \& A. R. SHERWOOD. 2002. Phylum Rhodophyta (Red Algae). In: The Freshwater Algal Flora of the British Isles: an identification guide to freshwater and terrestrial algae. D. M. John, B. A. Whitton \& A. J. Brook. (eds.): 123143. Cambridge University Press, Cambridge.

STARMACH, K. 1966. Homoeothrix crustacea Woronichin i glony towarzyszace $\mathrm{w}$ górnym biegu Raby. Acta Hydrobiologica, 8: 309-320.

STARMACH, K. 1977. Phaeophyta-Brunatnice and Rhodophyta-Krasnorosty. Flora Slodkowodna Polski 14. Ed. Polska, Academia, Warszawa.

TOMÁS, X. 1981. Thorea ramosissima en un canal del litoral Valenciano. Folia Botanica Miscellanea, 2: 71-74.

TOMÁS, X., P. LÓPEZ, R. MARGALEF \& F. A. COMÍN. 1980. Distribution and ecology of Compso- 
pogon coeruleus (Balbis) Montagne (Rhodophyta, Bangiophyceae) in Eastern Spain. Cryptogamie Algologie, 1(3): 179-186.

VAN DEN HOEK, C., D. G. MANN \& H. M. JAHNS. 1995. Algae. An Introduction to Phyco- logy. Cambridge University Press, Cambridge. WOLOWSKI, K., J. KOWALSKA, \& F. HINDÁK. 2007. Chroodactylon ornatum (Rhodophyta, Porphyridiales) occurring in Poland and Slovakia. Biologia Bratislava, 62(6): 646-649. 